\title{
Capacidad de carga turística y zonificación del CEANA, con miras al manejo de visitantes y protección del recurso hídrico en Cartago, Costa Rica
}

\author{
Tourism carrying capacity and environmental zoning of CEANA, oriented to visitors \\ management and water resource protection in Cartago, Costa Rica
}

Leonardo Castro-Barrantes ${ }^{1}$

Recibido: 28/11/2019

Aceptado: 31/03/2020

Publicado: 28/07/2020

\begin{abstract}
The Municipality of Cartago implemented by the end of the 2019 the Complejo Educativo Ambiental Naciente Arriaz (CEANA) as an innovative project oriented to environmental education for the community and aimed to become a model of sustainable practices that can be carried out to contribute to water protection. CEANA is located at a fragile ecosystem where water is collected from a spring and it is distributed to the surrounding population, so there is a need to generate information and management instruments aimed at its long-term conservation as well as look for the protection of its water resource. The objective of this research was to determine the tourism carrying capacity of its three visitor's trails and establish the environmental zoning of the natural space. A forest inventory was carried out, it was performed a topographic survey on the three trails and high-resolution images of the area were taken with a drone. The maximum limit of daily visitors for Puente de Arco's trail, Naciente Arriaz's trail and Bosque en Regeneración's trail were 31, 26 and 56 people, respectively. The environmental zoning of CEANA consisted of five management zones: low intervention zone, medium intervention zone, high intervention zone, natural regeneration zone and buffer zone.
\end{abstract}

Key words: Naciente Arriaz, tourism carrying capacity, environmental zoning, trails. 


\section{Resumen}

La Municipalidad de Cartago implementó a finales del 2019 el Complejo Educativo Ambiental Naciente Arriaz (CEANA) como un innovador proyecto orientado a la educación ambiental y enfocado en la conservación del recurso hídrico y la naturaleza. El CEANA se encuentra ubicado en un ecosistema ambientalmente frágil y donde se capta agua de una naciente para la población aledaña, por lo que surge la necesidad de generar información e instrumentos de manejo orientados a la conservación a largo plazo y enfocados en proteger el recurso hídrico. El objetivo de esta investigación fue determinar la capacidad de carga turística de los tres senderos de visitación y establecer la zonificación ambiental del espacio natural. Se realizó un inventario forestal en el bosque, se levantaron topográficamente los senderos y se tomaron fotografías aéreas de alta resolución del área de estudio con un dron. Se determinó que el límite máximo de visitantes diarios para los senderos Puente de Arco, Naciente Arriaz y Bosque en Regeneración fue de 31, 26 y 56 personas, respectivamente. La zonificación del CEANA consistió en cinco zonas para el manejo: zona de baja intervención, zona de mediana intervención, zona de alta intervención, zona de regeneración natural y zona de amortiguamiento.

Palabras clave: Naciente Arriaz, capacidad de carga turística, zonificación ambiental, senderos de visitación.

\section{Introducción}

El informe de las Naciones Unidas del año 2015 sobre los recursos hídricos en el mundo [1], prevé que para el año 2030 el planeta tendrá que enfrentarse a un déficit mundial del $40 \%$ de agua. A pesar de que Costa Rica es considerado un país rico en este recurso (31300 m3/persona/año), se estima que el $75 \%$ de las fuentes de abastecimiento están calificadas como altamente vulnerables [2]. Además, este recurso no se encuentra bien distribuido geográfica ni estacionalmente en el país, por lo que la falta de ordenamiento territorial, los cambios de uso del suelo, el deterioro de las cuencas hidrográficas y la contaminación de las fuentes de agua amenazan su potencial [3].

La gestión integral del recurso hídrico se ha convertido en el paradigma actual de la gestión del agua a nivel mundial [4], con el fin de administrarlos en forma sostenible y equilibrada, considerando intereses sociales, económicos y ambientales, bajo el imprescindible actuar de gobiernos e instituciones [5]. Esta gestión implica también comprender la relación entre las necesidades humanas y del ecosistema dentro de una cuenca hidrográfica [6]. La necesidad de proporcionar agua limpia con un presupuesto limitado, obliga a muchos municipios y gobiernos locales a estudiar enfoques innovadores a fin de mantener suministros de agua potable a los asentamientos urbanos [7].

Para el año 2019, la Municipalidad de Cartago propone un innovador proyecto denominado Complejo Educativo Ambiental Naciente Arriaz (CEANA). El CEANA se ubica en un área boscosa que resguarda la naciente Arriaz, la cual abastece de agua potable a los poblados de Taras, La Lima y parte de La Fátima [8]. Este complejo educativo fue creado con la intención de poner fin a los problemas de vandalismo y contaminación ocurridos en el área de la naciente y contrarrestarlos con el establecimiento de un nuevo programa de educación ambiental para la comunidad enfocado en la conservación del recurso hídrico [9]. El sitio cuenta con amplio espacio e infraestructura para llevar a cabo las labores de educación ambiental: aula para talleres, charlas e implementación de programas didácticos, oficinas administrativas, pequeñas áreas demostrativas sobre buenas prácticas agrícolas y áreas de juegos y recreación. Además, el sitio posee tres senderos de interpretación dentro del bosque, los cuales cuentan con 16 estaciones interpretativas que poseen información didáctica y apoyan las visitas guiadas realizadas por un educador ambiental que siempre debe acompañar los visitantes. De esta manera, se conforma un complejo educativo que se plantea el objetivo de desarrollar e implementar acciones dirigidas a la protección del recurso hídrico en el cantón de Cartago, potenciado a la vez su desarrollo socioeconómico [10].

Al tratarse de una zona de alta importancia y vulnerabilidad hídrica, el CEANA solo permite visitas a grupos de personas previamente agendados y con un estricto control de la administración, que evita el ingreso al público en general y personas que no cuenten con reservación. Sin embargo, también surge la necesidad de generar instrumentos de manejo del espacio natural, orientados a la conservación a largo plazo de sus recursos naturales. El presente estudio tiene como objetivos determinar la capacidad de carga de los tres senderos de visitación y desarrollar la zonificación ambiental del CEANA, de manera que permita gestionar el uso del espacio y el ordenamiento de las actividades dentro del espacio natural.

\section{Materiales y métodos}

\section{Área de estudio}

EI CEANA posee una extensión de 4,31 hectáreas y se ubica a una altitud promedio de $1525 \mathrm{msnm}$ en el poblado 
de Quircot, provincia de Cartago, Costa Rica (punto medio en las coordenadas geográficas $9^{\circ} 53^{\prime} 21.86^{\prime \prime} \mathrm{N}$; $83^{\circ} 56^{\prime} 5.32^{\prime \prime}$ ) (Figura 1). Dentro de su área se ubica la naciente Arriaz, la cual posee una producción de $60 \mathrm{~L} / \mathrm{s}$ [8] y se encuentra totalmente protegida por una estructura de concreto, donde parte de su caudal es captado por la Municipalidad de Cartago para el abastecimiento de las comunidades aledañas. La presencia de la Naciente Arriaz hace que el área de estudio se ubique en una zona de protección por naciente [11], y también en una zona de protección absoluta del área de recarga acuífera, según lo señalan estudios técnicos de [12].

Según la clasificación de [13], la zona de vida presente es Bosque Húmedo Premontano (bh-P) y según datos del [14], para la región se reporta una temperatura media anual entre los $18-20^{\circ} \mathrm{C}$ y una precipitación anual que oscila entre los 1400-2000 mm.

\section{Recolección de datos}

Se realizó un inventario forestal con una intensidad de muestreo de 11,6 \% para diagnosticar la composición y estructura horizontal del bosque del CEANA. Se levantaron cinco parcelas rectangulares de $20 \times 50$ metros $(0,1 \mathrm{ha})$, las cuales fueron seleccionadas aleatoriamente. En cada parcela se midió el diámetro a 1,30 metros del suelo y se identificó la especie para todos los individuos mayores a $10 \mathrm{~cm}$ de diámetro. Para la determinación de la capacidad de carga turística, cada uno de los senderos fue levantado topográficamente y se cronometró el tiempo del recorrido. Posteriormente, se sistematizó la información de campo y se ubicaron los senderos espacialmente a través de SIG. Para la zonificación del complejo, se realizó un vuelo con un vehículo aéreo no tripulado (Drone modelo DJI Phantom 4 Pro) y se capturaron fotografías aéreas de alta resolución del área de estudio.

\section{Cálculo de capacidad de carga turística}

La metodología utilizada para determinar la capacidad de carga turística de los senderos fue adaptada de [15] y [16]. Consta de tres niveles: capacidad de carga física (CCF), capacidad de carga real (CCR) y capacidad de carga efectiva (CCE).

\section{Capacidad de carga física (CCF)}

Corresponde al límite máximo de visitas permitidas en un espacio definido y tiempo determinado. Para su cálculo se utilizó la ecuación 1.

$$
C C F=\frac{S}{s p} * N V
$$

Donde $S$ corresponde a la longitud del sendero $(\mathrm{m})$ y $s p$ a la longitud utilizada por persona (1 metro lineal). $N v$ corresponde al número de veces que el sitio puede ser visitado por la misma persona en un día (visitas/día/ visitante) y se representa por la relación $\mathrm{NV}=\mathrm{Hv} / \mathrm{tv}$ (donde $H v$ es el horario de visitación del CEANA en horas por día y $t v$ es el tiempo necesario para recorrer el sendero en horas).

\section{Capacidad de carga real (CCR)}

Es el límite máximo de visitas que puede recibir un sendero condicionado a sus limitantes ambientales. Está determinada a partir de la CCF, luego de ser sometida a factores de corrección definidos en función de las características particulares del sendero. Los factores de corrección considerados en este estudio fueron: vulnerabilidad hídrica, erodabilidad, accesibilidad, precipitación y cierres temporales. La capacidad de carga real se calculó a través de la ecuación 2.

CCR $=$ CCF $($ FCvh $*$ FCero $*$ FCacc $*$ FCpre $*$ FCtem $)(2)$

Donde, CCF corresponde a capacidad de carga física (visitas/día), FCvh corresponde al factor de corrección vulnerabilidad hídrica, FCero al factor de erodabilidad, FCacc al factor de accesibilidad, FCpre factor de precipitación y FCtem corresponde al factor cierres temporales.

Los factores de corrección FCvh, FCero y FCacc fueron determinados a partir de la ecuación 3.

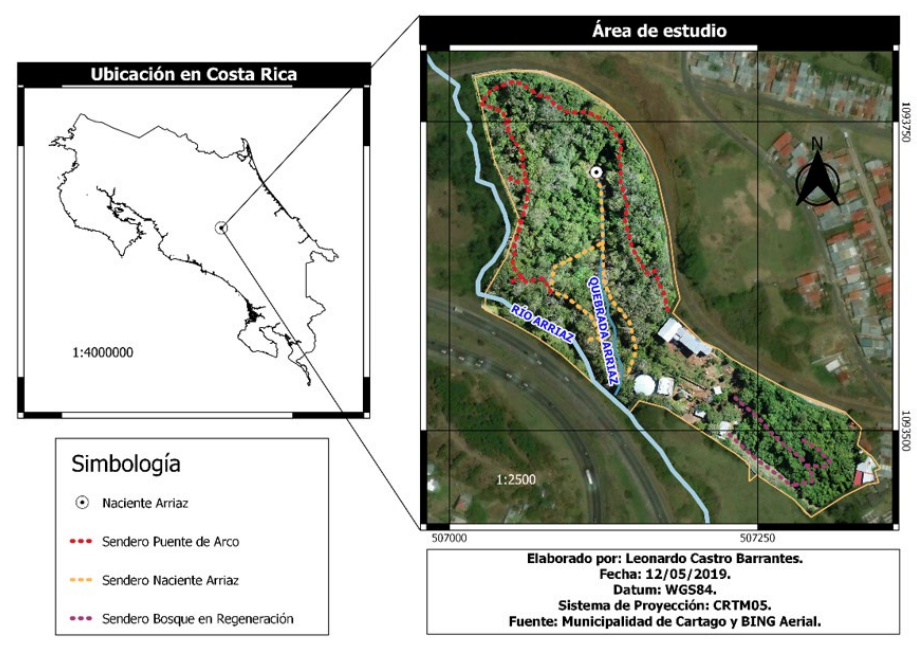

Figura 1. Ubicación geográfica del CEANA.

Figure 1. Geographic location of CEANA. 


$$
F C=1-\frac{((m a * 1.5)+(m m * 1))}{m t}
$$

Donde, FC corresponde al factor de corrección; ma corresponde a los metros de sendero ubicados en zonas con alta vulnerabilidad hídrica, alto riesgo de erodabilidad o bien alta dificultad de accesibilidad; $\mathrm{mm}$ corresponde a los metros de sendero ubicados en zonas con vulnerabilidad hídrica media, riesgo de erodabilidad media o con dificultad de accesibilidad media; y $\mathrm{mt}$ corresponde a los metros totales del sendero.

Los factores de corrección FCpre y FCtem se determinaron a partir de la ecuación 4.

$$
F C=1-\frac{h l}{h t}(4)
$$

Donde, FC corresponde al factor de corrección; $h l$ corresponde a las horas de lluvia limitantes al año o las horas al año en que el CEANA permanece cerrado fuera de su horario normal; $y$ ht corresponde a las horas al año que el CEANA permanece abierto para la visitación de sus senderos.

\section{Capacidad de manejo (CM)}

Considera variables administrativas, logísticas e insumos que permiten el desarrollo de las actividades y el cumplimiento de los objetivos dentro del espacio natural. En este estudio se consideraron las variables infraestructura, equipo y personal. Cada variable se calificó bajo cuatro criterios (cantidad, estado, localización y funcionalidad), excepto la variable personal, la cual sólo se calificó según cantidad.

Cada criterio fue calificado por la administración del CEANA, según el Cuadro 1.

Cuadro 1. Escala de calificación adaptada de la norma ISO 10004.

Table 1. Qualification scale adapted from ISO 10004

\begin{tabular}{|ccc|}
\hline Porcentaje (\%) & Valor & Calificación \\
\hline$\leq 35$ & 0 & Insatisfactorio \\
\hline $36-50$ & 1 & Poco satisfactorio \\
\hline $51-75$ & 2 & $\begin{array}{c}\text { Medianamente } \\
\text { satisfactorio }\end{array}$ \\
\hline $76-89$ & 3 & Satisfactorio \\
\hline$\geq 90$ & 4 & Muy satisfactorio \\
\hline
\end{tabular}

El cálculo de la CM se realizó a partir de un promedio de los factores de las tres variables, expresado en porcentaje a través de la ecuación 5 .

$$
C M=\frac{(I+E+P)}{3}
$$

Donde CM corresponde a capacidad de manejo (\%), I corresponde al factor obtenido de la evaluación de la infraestructura, $E$ al factor obtenido de la evaluación del equipo y $P$ al factor obtenido de la evaluación del personal.

\section{Capacidad de carga efectiva (CCE)}

Se obtuvo a partir de la ecuación 6. Donde CCR corresponde a la capacidad de carga real (visitas/día) y $\mathrm{CM}$ a la capacidad de manejo.

$$
C C E=C C R * \frac{C M}{100}(6)
$$

El número máximo de visitas por día se determinó mediante la ecuación 7. Donde, CCE corresponde a la capacidad de carga efectiva (visitas/día) y $N V$ al número de veces que el sitio puede ser visitado por la misma persona en un día (visitas/día/visitante).

$$
\text { Visitas } / \text { día }=\frac{C C E}{N V}(7)
$$

\section{Zonificación ambiental}

Se realizó delimitando el área de estudio en Unidades Ecológicas del Paisaje (UEP) [17], de manera que pudieran ser agrupadas en zonas acordes con los objetivos de conservación y educación ambiental que plantea el CEANA. El método utilizado se basó en las propuestas de zonificación de [17] y [18], las cuales se adaptaron al nivel de microzona. El criterio para definir las UEP fue el tipo de cobertura actual, pues permite aprovechar los contornos naturales del paisaje en las transiciones de uso del suelo. Para determinar el tipo de cobertura se utilizó la información obtenida del inventario forestal y se analizó espacialmente la ortofoto a través de herramientas de SIG.

Las zonas que fueron consideradas para el uso y manejo del CEANA se seleccionaron con base en las recomendaciones propuestas por [19] para la zonificación en áreas protegidas. Además, se consideró una zona de amortiguamiento entre el territorio propiedad del CEANA y los terrenos colindantes de propiedad privada. Para cada zona se realizó una descripción, se establecieron 
objetivos de conservación y desarrollo, se sugirieron prácticas de manejo y se indicaron actividades no recomendadas.

\section{Análisis de datos}

Se determinó el área basal por hectárea $\left(\mathrm{m}^{2} / \mathrm{ha}\right)$ para el bosque del CEANA y el índice de valor de importancia (IVI) para cada especie muestreada [20]. Se utilizó el software Agisoft PhotoScan para generar una ortofoto que pudiera ser utilizada como insumo en la zonificación y el software QGIS versión 3.6 para crear la cartografía temática, el mapa de zonificación y determinar el área de cada zona de manejo. Toda la sistematización de información, análisis y cálculo de factores se realizó con hojas electrónicas de Microsoft Excel.

\section{Resultados y discusión}

\section{Composición florística y estructura horizontal del bosque}

El bosque del CEANA posee una riqueza de 28 especies (individuos $\geq 10 \mathrm{~cm}$ de diámetro). El cuadro 2 muestra las cinco principales especies arbóreas en el ranking de índice de valor de importancia (IVI).

El bosque está compuesto por dos estratos; el primero de ellos, con un área aproximada de 2,92 ha, posee un área basal de $28,19 \mathrm{~m}^{2} / \mathrm{ha}$ y corresponde a bosque secundario de estadio tardío. El segundo estrato corresponde a una pequeña área en regeneración $(0,74$ ha) de bosque secundario estadio intermedio, el cual posee un área basal de $14,52 \mathrm{~m}^{2} /$ ha y se caracteriza por una gran abundancia de especies pioneras de la familia Asteraceae, sin embargo, se encuentran establecidas algunas heliófitas durables que empiezan a cerrar el dosel (Inga punctata Willd, Ficus pertusa L. f. y Casimiroa edulis La Llave \& Lex.). Ambos estratos poseen un área basal similar a lo reportado por [21] para bosques secundarios húmedos premontanos y su composición de especies como Quercus insignis M. Martens \& Galeotti, Sideroxylon persimile (Hemsl.), Stemmadenia litoralis (Kunth) L. Allorge y Cedrela tonduzii C. DC, representan los muy escasos parches de bosque húmedo premontano que persisten en el país y las especies autóctonas del Valle Central [22], [23].

Ambos fragmentos boscosos del CEANA son producto del proceso de sucesión natural y la intervención humana que ha recibido el área. Ciertos factores como el histórico uso de la naciente Arriaz para el abastecimiento de agua y la matriz de uso del suelo que se ha desarrollado en la periferia del CEANA (asentamientos urbanos, cultivos agrícolas, industrias y la carretera Florencio del Castillo), han influenciado en la dinámica del bosque al convertirlo en un fragmento con poca conectividad. Las fuentes semilleras provenientes de otros fragmentos de bosque y los arboles remanentes presentes son elementos que influyen en la diversidad de un ecosistema secundario [24], sin embargo, otros factores como el historial de intervención y uso del suelo, han sido los de mayor influencia en la composición de estos fragmentos; esto se evidencia con la dominancia y abundancia de la especie introducida Fraxinus uhdei (Wenz.) Lingelsh, que se encuentra en la primera posición del ranking de IVI (Cuadro 2), patrón similar al encontrado por [25] en fragmentos de bosque secundario ubicados en Dulce

Cuadro 2. Principales especies arbóreas según índice de valor de importancia (IVI) en cinco parcelas de 0,1 ha ubicadas en el bosque del CEANA, Cartago, Costa Rica.

Table 2. Main tree species according to importance value index (IVI) in five plots of 0.1 ha located in CEANA's forest, Cartago, Costa Rica.

\begin{tabular}{|c|c|c|c|c|c|c|c|}
\hline \multirow{2}{*}{ Especie } & \multicolumn{2}{|c|}{ Abundancia } & \multicolumn{2}{|c|}{ Frecuencia } & \multicolumn{2}{|c|}{ Dominancia } & \multirow{2}{*}{ IVI } \\
\hline & Abs & $A \%$ & Abs & $\mathrm{F} \%$ & Abs & $\mathrm{D} \%$ & \\
\hline Fraxinus uhdei & 28 & 14,74 & 5 & 8,20 & 4,72 & 37,10 & 60,03 \\
\hline Quercus insignis & 26 & 13,68 & 4 & 6,56 & 1,90 & 14,92 & 35,17 \\
\hline Sideroxylon persimile & 20 & 10,53 & 3 & 4,92 & 1,58 & 12,40 & 27,85 \\
\hline Inga punctata & 23 & 12,11 & 5 & 8,20 & 0,60 & 4,74 & 25,04 \\
\hline Stemmadenia litoralis & 15 & 7,89 & 4 & 6,56 & 0,53 & 4,20 & 18,65 \\
\hline Subtotal 5 especies & 112 & 58,95 & 21 & 34,43 & 9,34 & 73,37 & 166,74 \\
\hline $\begin{array}{l}\text { Subtotal restante de } \\
\text { especies }\end{array}$ & 78 & 41,05 & 40 & 65,57 & 3,39 & 26,63 & 133,26 \\
\hline Total & 190 & 100 & 61 & 100 & 12,73 & 100 & 300 \\
\hline
\end{tabular}


Cuadro 3. Capacidad de carga turística de los senderos Puente de Arco, Naciente Arriaz y Bosque en Regeneración, CEANA, Cartago, Costa Rica.

Table 3. Tourism carrying capacity for Puente de Arco, Naciente Arriaz and Bosque en Regeneración trails, CEANA, Cartago, Costa Rica.

\begin{tabular}{|c|c|c|c|}
\hline \multirow{2}{*}{ Capacidad de carga } & \multicolumn{3}{|c|}{ Senderos } \\
\hline & Puente de Arco & Naciente Arriaz & Bosque en Regeneración \\
\hline $\begin{array}{c}\text { Capacidad de carga física } \\
\text { (visitas/día) }\end{array}$ & 3058 & 2386 & 2760 \\
\hline Factor vulnerabilidad hídrica & 0,46 & 0,16 & 1 \\
\hline Factor erodabilidad & 0,46 & 1 & 0,62 \\
\hline Factor accesibilidad & 0,46 & 0,89 & 0,62 \\
\hline Factor precipitación & 0,85 & 0,85 & 0,85 \\
\hline Factor cierres temporales & 0,97 & 0,97 & 0,97 \\
\hline $\begin{array}{c}\text { Capacidad de carga real } \\
\text { (visitas/día) }\end{array}$ & 245 & 238 & 874 \\
\hline $\begin{array}{l}\text { Calificación variable } \\
\text { infraestructura }\end{array}$ & 0,96 & 0,96 & 0,96 \\
\hline Calificación variable equipo & 0,93 & 0,93 & 0,93 \\
\hline Calificación variable personal & 0,41 & 0,41 & 0,41 \\
\hline Capacidad de manejo & $77 \%$ & $77 \%$ & $77 \%$ \\
\hline $\begin{array}{c}\text { Capacidad de carga efectiva } \\
\text { (visitas/día) }\end{array}$ & 188 & 187 & 649 \\
\hline NV & 6 & 7 & 12 \\
\hline Visitantes/día & 31 & 26 & 56 \\
\hline Visitantes/año & 7440 & 6240 & 13440 \\
\hline
\end{tabular}

Nombre de Cartago. Este comportamiento demuestra la intervención que ha tenido el sitio, pues la especie fue plantada homogéneamente por toda el área, sin embargo, dadas las condiciones de uso del suelo colindantes y la fragilidad hídrica a las que está expuesta la naciente Arriaz, esta especie forma parte de un bloque boscoso que ha desempeñado un papel fundamental en los procesos de reducción de escorrentía superficial, retención de sedimentos, infiltración y protección de los cuerpos de agua del ecosistema [26].

\section{Capacidad de carga turística de los senderos}

La CCF obtenida indica que el límite máximo de visitas, considerando solamente el espacio disponible, el tiempo de visita y la necesidad de espacio por persona, es de 3058, 2386 y 3760 visitas/día para los senderos Puente de Arco (530 m de largo), Naciente Arriaz (342 $\mathrm{m}$ de largo) y Bosque en Regeneración (230 m de largo), respectivamente (Cuadro 3). A pesar de la CCF ser muy específica de cada sitio y dependiente del NV, como de la longitud del sendero, investigaciones afines realizadas en senderos con longitudes menores a 1 kilómetro obtuvieron valores similares de CCF [27], [28].
Referente a los valores de CCR, los resultados indican que los senderos con mayores limitantes ambientales corresponden al sendero Puente de Arco (CCR de 245 visitas/día) y el sendero Naciente Arriaz (CCR de 238 visitas/día) (Cuadro 3). Los factores que más restringen al sendero Puente de Arco son el FCero $(0,46)$ y FCacc $(0,46)$, valores explicados por la presencia de pendientes escarpadas en algunos tramos del sendero, además del FCvh $(0,46)$ que también restringe en mayor medida la visitación del sendero Naciente Arriaz $(\mathrm{FCvh}=0,16)$; esto se debe a la condición que poseen ambos senderos de estar ubicados en áreas colindantes o muy cercanas a los cuerpos de agua del CEANA. Por el otro lado, el sendero Bosque en Regeneración posee una mayor capacidad de carga real (874 visitas/día), pues posee pendientes poco escarpadas y el FCvh es de 1 ya que no hay tramos de sendero situados en zonas de vulnerabilidad hídrica.

A pesar de haberse realizado estudios de capacidad de carga en áreas protegidas de alta importancia hídrica [29], [30] y/o destinadas a la producción de agua para consumo humano combinadas con ecoturismo [31], no se ha reportado la aplicación de un factor limitante que restrinja el número de visitantes debido a la vulnerabilidad hídrica a la que se encuentran expuestas quebradas, ríos 
y/o nacientes por la permanencia y tránsito de personas en áreas de uso público como senderos colindantes a los cuerpos de agua. Cualquier actividad realizada al interior o adyacente a la zona ribereña, como el mantenimiento de caminos o senderos y el desarrollo recreacional, tiene el potencial de impactar negativamente la calidad del agua, el hábitat terrestre y acuático, y las funciones ecológicas de las franjas ribereñas [26], [32]; esto justifica el diseño e implementación del FCvh. Este factor intenta controlar los impactos producidos por el tránsito de personas en lo senderos a través de una disminución en la visitación, considerando los efectos e impactos causados por la actividad turística o recreacional hacia el agua, basándose en las perspectivas y dimensiones ecológicas de la capacidad de carga [33].

La capacidad de manejo obtenida es de $77 \%$, determinando que la CCE para los senderos Puente de Arco, Naciente Arriaz y Bosque en Regeneración, es de 188, 187 y 649 visitas por día, respectivamente (Cuadro 3). Este valor de $\mathrm{CM}$ se considera como una calificación "satisfactoria" según los criterios de evaluación de la capacidad de manejo (Cuadro 1) e implica que el CEANA tiene condiciones muy aceptables para recibir visitantes, pero pueden ser mejoradas para que sean las óptimas [16]. A pesar de que las variables infraestructura y equipo obtuvieron valores "muy satisfactorios" de 0,96 y 0,93 respectivamente (Cuadro 3 ), la variable personal obtuvo una calificación "poco satisfactoria" de 0,41 atribuida al faltante de recurso humano principalmente en labores de educación ambiental y gestión del área natural. Si el CEANA tuviera un mayor número de educadores ambientales (actualmente se limita a una persona) se podrían dirigir varios grupos en los senderos simultáneamente, lo que permitiría un mayor ingreso de visitantes; además, si se contase con más profesionales encargados de la gestión del área natural se podría tener un mayor control en la ejecución de las actividades de manejo planteadas para cada zona del CEANA y así, se abarcarían más espacios educativos para una recepción de grupos mayores. Estudios como los de [34] realizados en la Reserva Natural Absoluta Cabo Blanco, donde se obtienen calificaciones poco satisfactorias de $\mathrm{CM}$, muestran que se reduce hasta en un $53 \%$ la CCR de los senderos; o en contraste, estudios como los [35] demuestran que la $\mathrm{CM}$ reduce solamente en un $12 \%$ la CCR de los senderos del Parque Ecológico Chipinque en México; esto demuestra la relevancia que posee la capacidad de manejo y sus variables como indicadores de gestión de un área natural y como su deficiencia afecta directamente la CCT de sus áreas de uso público [36].

En cuanto al número máximo de visitantes por día (Cuadro 3), se obtiene que deberían admitirse 31 visitantes/día para el sendero Puente de Arco, 26 visitantes/día para el sendero Naciente Arriaz y 56 visitantes/día para el sendero Bosque en Regeneración.
Estos valores permiten aproximar la intensidad de uso de los senderos, adaptando el desarrollo de las actividades de educación ambiental en los senderos según las capacidades del ecosistema [15], [37], [38]. Además, son funcionales, pues, si se restringe la visitación de todos los senderos al menor valor de CCT (26 visitantes/día del sendero Naciente Arriaz), la administración del CEANA y el educador ambiental podría manejar un grupo de esa magnitud más eficientemente dada sus limitantes de recurso humano evidenciadas en la $\mathrm{CM}$, y puede permitir a los visitantes tener una experiencia de mayor calidad. Adicionalmente, si se adopta una CCT de 26 visitantes/ día en todos los espacios de uso público del CEANA, se está facilitando su gestión, tomando en consideración que la entrada de visitantes al complejo y sus senderos está condicionada a que solamente ingresen grupos previamente agendados y siempre en compañía de un educador ambiental. A pesar de no hallarse estudios de CCT en áreas naturales con características similares al CEANA en cuanto a gestión y reglas de ingreso de visitantes, si se compara con estudios como los de [39], donde se calcula la CCT para un sendero situado en una finca pedagógica con objetivos educativos similares, se obtiene datos más conservadores (6 visitas/día) que los obtenidos en este estudio, evidenciando que la CCT es muy específica de las características espaciales, ambientales y de gestión de un área natural.

\section{Zonificación ambiental}

Se determinaron cinco zonas para el manejo del CEANA: zona de baja intervención, zona de mediana intervención, zona de alta intervención, zona de regeneración natural y zona de amortiguamiento (Figura 2). Un estudio realizado por [40] en un área protegida municipal plantea una delimitación de zonas con objetivos educativos y de conservación similares dirigidos hacia la población local; estas zonas buscan asegurar que los impactos, el uso del espacio y el ordenamiento de las actividades dentro del CEANA sea congruente con el mantenimiento de la integridad ecológica de la zona y sus servicios ecosistémicos [19].

Las actividades de manejo del área natural del CEANA están funcionalmente relacionadas al tipo de ecosistema de cada zona y direccionadas a cumplir cada objetivo de conservación y desarrollo, considerando también las restricciones legales que posee el sitio, pues el CEANA se encuentra situado sobre una zona de protección que impide la remoción de cobertura forestal [11]. Adicionalmente, se consideraron las capacidades administrativas del CEANA determinadas en los estudios de CCT, donde es importante tomar en cuenta que el recurso humano puede llegar a ser una limitante en la ejecución y control de las actividades de manejo en cada 
una de las zonas, como lo demuestran los resultados de CM del área natural (Cuadro 3).

\section{Zona de baja intervención}

La zona de baja intervención abarca un $57,2 \%$ del área del CEANA (2,46 ha), se ubica al noroeste y corresponde al primer estrato de bosque. Dadas las condiciones de fragilidad del ecosistema, esta zona debe ser la de menor intervención y máxima conservación. Se establecen como objetivos de conservación y desarrollo: proteger el área de recarga acuífera de la naciente Arriaz y conservar el relicto de bosque húmedo premontano, sus ecosistemas y especies de flora y fauna. Se promueven prácticas de reforestación con objetivos de restauración ecológica en zonas degradadas, de manera que propicien la recarga hídrica. El ingreso de personas a la zona sin la previa autorización de la administración del CEANA debe prohibirse, ni se debe permitir la construcción de edificaciones, caminos o alguna otra infraestructura que impermeabilice el suelo e implique remoción de la cobertura forestal.

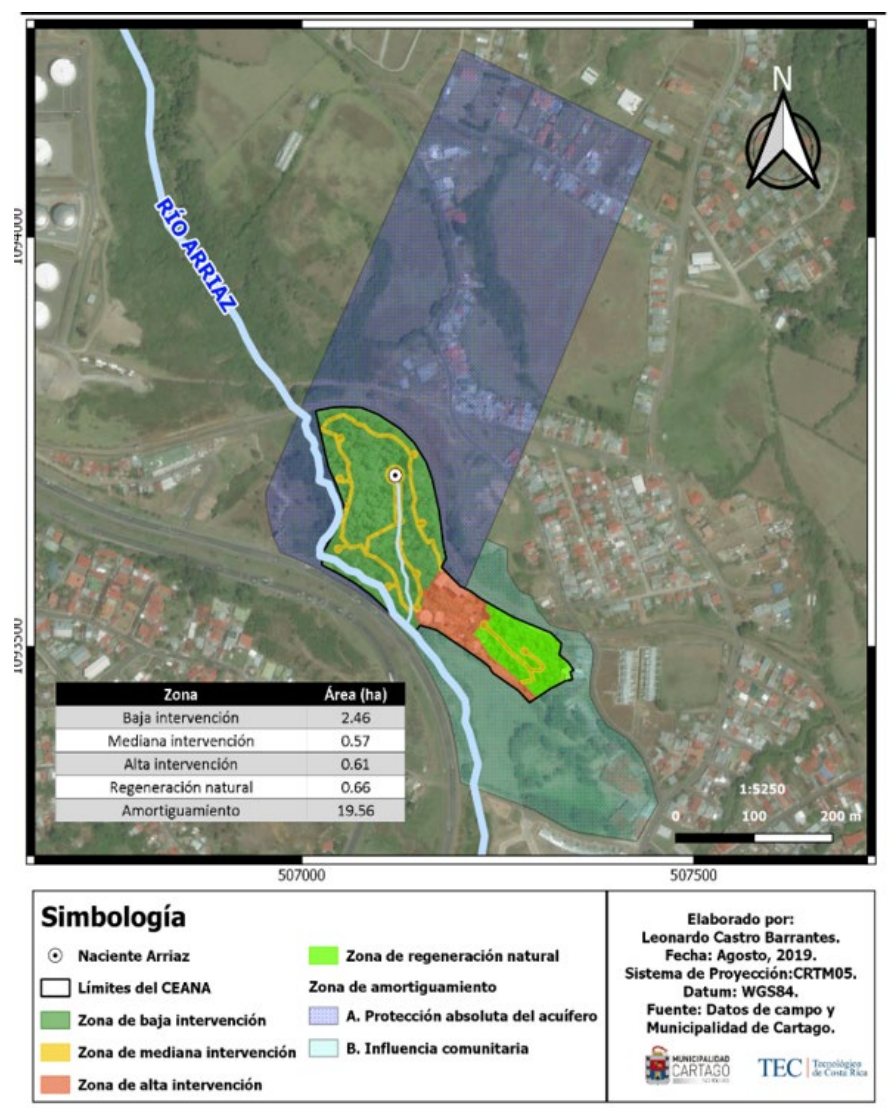

Figure 2. Zonificación ambiental del CEANA.

Figure 2. Environmental zoning for CEANA.

\section{Zona de mediana intervención}

Esta zona abarca un 13,3\% del área del CEANA $(0,57$ ha) y corresponde al área de desplazamiento de los transeúntes en los tres senderos y en sus respectivas estaciones de interpretación. Además, toma en cuenta el área de captación, la cual se encuentra sellada totalmente por una estructura de concreto, y todo su recorrido a través de la tubería hasta llegar al tanque de almacenamiento. Se establecen como objetivos de conservación y desarrollo: realizar actividades de educación ambiental a través de caminatas por los senderos interpretativos. Se promueve realizar prácticas de manejo orientadas al mantenimiento de los senderos y que garanticen la seguridad de los transeúntes, como podas y/o remoción de árboles muertos que presenten indicios de riesgo de caída hacia los límites del sendero; esto siempre bajo criterios técnicos y de sostenibilidad. Además, es adecuado la rotulación de especies presentes en los recorridos que presenten una singularidad intrínseca o importancia ecológica, de manera que los visitantes puedan tener un mayor conocimiento de las especies y su papel en el ecosistema. No se permite el ingreso a los senderos sin la respectiva autorización de la administración del CEANA y/o compañía del guía educador ambiental.

\section{Zona de alta intervención}

Esta zona abarca el 14,2 \% del área del CEANA (0,61 ha). Corresponde a un área modificada ecológicamente, donde no existe cobertura forestal significativa más que algunos árboles aislados. Aquí se encuentra el área para el recibimiento de los grupos de visitantes: aulas, oficinas administrativas, áreas de recreación, zona de buenas prácticas agrícolas, servicios sanitarios, entre otros. Se establecen como objetivos de conservación y desarrollo: administrar el CEANA y facilitar el desarrollo de actividades de educación ambiental, recreación y prácticas sostenibles. Se promueven actividades recreativas y educativas en las áreas e infraestructuras destinadas para este fin y no se permite el ingreso al público en general.

\section{Zona de regeneración natural}

Abarca un 15,3\% del área de estudio (0,66 ha). Se ubica en la región este del CEANA y corresponde al segundo estrato del bosque. Esta zona es provisional, pues una vez avanzada su regeneración natural hacia un estadio de sucesión de mayor madurez, pasará a ser parte de la zona de baja intervención. Se establecen como objetivos de conservación y desarrollo: promover el crecimiento y la regeneración natural del bosque secundario y proteger la biodiversidad de ecosistemas, flora y fauna que alberga esta zona de regeneración. Se promueve prácticas silviculturales como el enriquecimiento de 
especies y la reforestación en zonas degradadas. No se permite el ingreso de personas a la zona sin la previa autorización de la administración del CEANA ni se permite la construcción de edificaciones, caminos o alguna otra infraestructura que impermeabilice el suelo e implique remoción de la cobertura forestal.

\section{Zona de amortiguamiento}

Esta se divide en dos subzonas: A) Protección absoluta del acuífero y B) Influencia comunitaria.

\section{Subzona A: Protección absoluta del acuífero}

En esta área se encuentran terrenos de propiedad privada ubicados sobre la zona de protección absoluta de recarga acuífera, la cual corresponde a 130 metros de ancho a cada lado y 660 metros de largo, a partir del tubo de flujo que influye en la descarga de la naciente [12]. Se caracteriza por un uso del suelo dominado por potreros, cultivos y viviendas, con la presencia de algunos escasos parches boscosos [9]; además, según los estudios de vulnerabilidad a la contaminación realizados por [12], la zona se clasifica como altamente vulnerable. Se establecen como objetivos de conservación y desarrollo: propiciar acciones dirigidas a la conservación, vigilancia, restauración de ecosistemas, capacitación de grupos y concientización de la comunidad sobre la importancia de proteger, resguardar y mantener libre de contaminantes la zona de protección absoluta del acuífero. Se promueve la elaboración de un plan de educación ambiental orientado a los vecinos, agricultores e industrias que poseen terrenos situados en esta zona y realizan actividades productivas que pueden poner en riesgo el acuífero. Según recomiendan los estudios técnicos de [12], en esta subzona sólo se debería permitir la reforestación con fines de protección al recurso hídrico y ningún otro tipo de actividad productiva debería ser realizada aquí; sin embargo, la mayoría de propiedades privadas poseen un uso de suelo que difiere de tener cobertura forestal significativa. Es por esto que resulta prioritario, diseñar e implementar una estrategia de permita reforestar linderos de fincas, zonas de protección o inclusive combinar la reforestación con actividades productivas de la región.

\section{Subzona B: Influencia comunitaria}

El área abarca la zona sur de franja ribereña del río Arriaz colindante a la autopista Florencio del Castillo, el condominio Villa Real, un lote donde se ubica la antigua fábrica de gelatinas, algunas casas al este del CEANA y una pequeña área colindante a un charral arbolado. Esta subzona se caracteriza por estar ocupada por asentamientos urbanos, los cuales tienen una influencia directa sobre el CEANA al ubicarse en sus bordes; por tanto, las prácticas ambientales realizadas por los ciudadanos vecinos incidirán sobre el espacio natural. Se establecen como objetivos de conservación y desarrollo: establecer una integración del CEANA con las comunidades aledañas mediante el desarrollo de actividades recreativas y de educación ambiental, y promover alianzas y prácticas ambientales sostenibles en las comunidades vecinas. Se sugiere diseñar un plan de reforestación en conjunto con la comunidad para la reforestación de especies nativas en la ribera del río Arriaz. Además, es recomendable elaborar un plan de educación ambiental comunitaria enfocado en prácticas sostenibles de manejo de desechos sólidos y conservación del recurso hídrico, así como actividades recreativas que involucren más la comunidad con el CEANA.

\section{Conclusiones}

Se determinó que los fragmentos boscosos del CEANA corresponden a bosque secundario en dos estadios de sucesión diferentes y su composición se ha visto influenciada por los procesos de regeneración natural, influencia de la matriz de uso del suelo colindante e histórico uso de la naciente Arriaz.

Los estudios de capacidad de carga turística señalaron que el límite máximo de visitantes diarios que deben ser permitidos para los senderos Puente de Arco, Naciente Arriaz y Bosque en Regeneración es de 31, 26 y 56 personas, respectivamente. Sin embargo, es de suma importancia considerar que la capacidad de carga es variable con el paso del tiempo, por lo tanto, estudios de capacidad de carga deben ser actualizados periódicamente.

Dadas las condiciones de vulnerabilidad a las que están expuestos los cuerpos de agua del CEANA, fue necesario la aplicación de un factor de corrección limitante que restringiera el número máximo de visitantes que pueden ingresar a sus senderos y así se intenten disminuir los impactos ambientales al recurso hídrico.

La zonificación del CEANA estableció gestionar el uso del espacio y el ordenamiento de las actividades a través de cinco zonas: 1) zona de baja intervención, 2) zona de mediana intervención, 3) zona de alta intervención, 4) zona de regeneración natural y 5) zona de amortiguamiento (la cual se subdivide en dos subzonas; a) protección absoluta del acuífero y b) influencia comunitaria).

La capacidad de manejo evidenció deficiencias en la cantidad de recurso humano disponible para cumplir las funciones de educación ambiental y manejo del área protegida, que se reflejan en una disminución de visitantes diarios permitidos y una posible limitante en la 
ejecución de las actividades de manejo planteadas para cada zona.

\section{Agradecimientos}

A Gustavo Rojas, Arianne Gómez y Julio Urbina del Área Técnica de Acueductos de la Municipalidad de Cartago, a Dorian Carvajal y Mario Guevara de la Escuela de Ingeniería Forestal del TEC, al Programa de Movilidad Estudiantil del Consejo Nacional de Rectores (CONARE) y al Dr. Raúl Postigo Vidal, coordinador de la Escuela Universitaria de Turismo de Zaragoza (ETUZ).

\section{References}

[1] UNESCO, "Informe de las naciones unidas sobre los recursos hídricos en el mundo 2015”, 2015 [En línea]. Disponible en: http://www.unesco.org/new/fileadmin/MULTIMEDIA HQ/SC/images/WWDR2015Facts_Figures_SPA_web.pdf. [Consultado 23 noviembre 2019].

[2] R. Valverde, "Disponibilidad, distribución, calidad y perspectivas del agua en Costa Rica", Revista de Ciencias Ambientales, vol. 45, no. 1, pp 5-12, Junio, 2013.

[3] D. Hidalgo, "Recurso Hídrico en Costa Rica", Diagnóstico de los recursos hídricos en América Latina, Pearson Education, 2013, pp. 220-253.

[4] Y. Martínez y V.M. Villalejo, "La gestión integrada de los recursos hídricos: una necesidad de estos tiempos", Ingeniería Hidráulica y Ambiental, vol. 39, no. 1, pp. 58-72, Enero, 2018.

[5] GWP (Global Water Partnership), Manual para la Gestión Integrada de Recursos Hídricos en Cuencas, Global Water Partnership and International Network of Watershed Organizations, 2009, pp. 112.

[6] E. Vargas y A. Marín, "Costa Rica demanda una gestión integral del recurso hídrico: escenario latinoamericano y del país", Revista Intersedes, vol. 17, pp. 1-26, Mayo, 2016.

[7] L.S. Hamilton, Los bosques y el agua, FAO, 2009, pp. 101.

[8] J.S. Chin, P. Aguilar, D. Vega y M. Pérez, "Descripción biofísica y resultados finales de la calidad del agua del agua de la microcuenca del río Arriaz, asociada con el segmento del río comprendido entre su nacimiento y la naciente Arriaz dentro de las instalaciones del acueducto municipal, en la provincia de Cartago", Informe de consultoría para el Área Técnica de Acueductos, Municipalidad de Cartago, pp. 47, 2017.

[9] A. Guzmán y H. A. Díaz, "Identificación de las áreas de recarga acuífera y zonas de protección de los manantiales del cantón central de Cartago", presentado en el IX Congreso Nacional de Ciencias, Cartago, Costa Rica, 2007.

[10] Municipalidad de Cartago, "Centro Educativo Ambiental Naciente Arriaz (CEANA", 2019 [En línea]. Disponible en http://ceana.muni-carta.go.cr/. [Consultado 25 marzo 2020].
[11] Leyes y Decretos, "Ley Forestal N7575”, Asamblea Legislativa, Costa Rica, 1996 [En línea]. Disponible en https://www. cne.go.cr/cedo_dvd5/files/flash_content/pdf/spa/doc387/ doc387-contenido.pdf. [Consultado 25 marzo 2020].

[12] V. Ramos y I. Vargas, "Delimitación de las zonas de protección de los manantiales del cantón central de Cartago", Informe de consultoría para el Área Técnica de Acueductos, Municipalidad de Cartago, pp. 47, 2001.

[13] L. Holdridge, Life zone ecology, Tropical Science Center, 1967, pp 206.

[14] INDER (Instituto Nacional de Desarrollo Rural de Costa Rica), "Región Central Caracterización del Territorio CartagoOreamuno-El Guarco-La Unión”, 2016 [En línea]. Disponible en: https://www.inder.go.cr/correque/Caracterizacionterritorio-Cartago-Oreamuno-El-Guarco-La-Union.pdf. [Consultado 23 noviembre 2019]

[15] M, Cifuentes, "Determinación de capacidad de carga turística en áreas protegidas", Informe Técnico No. 194, CATIE, Turrialba, Costa Rica, 1992.

[16] M. Cifuentes, C. Mezquita, J. Méndez, M. Morales, N. Aguilar, D. Cancino, M. Gallo, M. Jolón, C. Ramírez, N. Ribeiro, E. Sandoval y M. Turcios, "Capacidad de Carga Turística de las áreas de uso público del Monumento Nacional Guayabo, Costa Rica". CATIE, 1999, pp. 75.

[17] A.L. Rodríguez, L. Lozano y P.C. Sierra, "Criterios de zonificación ambiental usando técnicas participativas y de información: estudio de caso zona costera del departamento del Atlántico", Boletín de Investigaciones Marinas y Costeras, vol. 41, no 1, pp. 61-83, 2012.

[18] R. Pinedo, "Zonificación como base para el ordenamiento territorial del municipio de Valle de Ángeles, Honduras", tesis de maestría, Centro Agronómico Tropical de Investigación y Enseñanza, Turrialba, Costa Rica, 2006.

[19] SINAC (Sistema Nacional de Áreas de Conservación). "Guía para el diseño y formulación del Plan General de Manejo de las Áreas Silvestres Protegidas de Costa Rica", San José, Costa Rica, 2013 [En línea]. Disponible en: http:// www.sinac.go.cr/ES/docu/ASP/Gu\%C3\%ADa\%20 dise \% C3\%B10\%20y\%20formulaci\%C3\%B3n\% 20 Plan\%20Manejo\%20ASP\%202013.pdf. [Consultado 23 noviembre 2019].

[20] J. Curtis \& R. Mclntosh, "An upland forest continuum in the prairie-forest border region of Wisconsin". Ecology, vol. 32, no 3, pp. 476-496, 1951.

[21] C.A. Ulate, "Análisis y comparación de la biomasa aérea de la cobertura forestal según zona de vida y tipo de bosque para Costa Rica", Tesis de Licenciatura, Instituto Tecnológico de Costa Rica, pp. 61, 2011.

[22] R. Quesada, "Los bosques de Costa Rica", IX Congreso Nacional de Ciencias, Instituto Tecnológico de Costa Rica, 2007 [En línea]. Disponible en http://www.cientec. or.cr/exploraciones/ponencias2007/RupertoQuesada.pdf. [Consultado 23 noviembre 2019].

[23] C. Rodríguez y L. Brenes, "Estructura y composición de dos remanentes de bosque premontano muy húmedo en la Reserva Madre Verde, Palmares, Costa Rica", Pensamiento Actual, vol. 9, no 12-13, pp. 115-124, 2009. 
[24] L. G. Acosta-Vargas, D. Reyes-Cordero, R. QuesadaMonge y M. Castillo-Ugalde, "Restauración del bosque seco tropical de Costa Rica: evolución del bosque y conservación de especies", 2012 [En línea]. Disponible en: http://copa.acguanacaste.ac.cr:8080/handle/11606/444 [Consultado 25 marzo 2020]

[25] O. Morales, "Caracterización florística y estructural de tres fragmentos boscosos secundarios en Cartago, Costa Rica", Biología Tropical, vol. 57, no 1, pp. 69-82, Noviembre, 2009.

[26] C. Cepeda y G. Navarro, "Protección del recurso hídrico en Costa Rica: propuesta para la reforma de los artículos 33 y 34 de la Ley Forestal", Serie Técnica, Boletín No. 95, CATIE, pp. 2-7, 2010.

[27] H.F. Soria, "Determinación de la capacidad de carga turística en los sitios de visita de la Reserva Nacional AllpahuayoMishana, Loreto-Perú", Ciencia Amazónica, vol 5, no 1, pp 10, abril, 2015.

[28] R.D. Ruiz-Acevedo, "Capacidad de carga turística del área de uso público del Parque Ecológico El Samán, Cartago, Valle", Tesis en Administración, Universidad Tecnológica de Pereira, Colombia, 2012.

[29] C. S. Vasconcelos y J.B.D. Câmara, "Evaluación de la capacidad de carga física del Parque Municipal de Itiquira, Formosa (GO), Brasil”, Estudios y perspectivas en turismo, vol. 21, no 4, pp. 996-1012, 2012.

[30] K, Cevallos, "Capacidad de carga turística del sendero Siete Cascadas como manejo sostenible del Área Provincial de Recreación Cerro de Hayas-Ecuador", Tesis de maestría, Universidad de Guayaquil, Ecuador, pp. 119, 2018.

[31] E, Maldonado y F. Montagnini, "Determinación de la capacidad de carga turística del Parque Nacional La Tigra Tegucigalpa, Honduras", Revista Forestal Centroamericana, vol. 34, pp. 47-51, Abril, 2001.

[32] M.J. Phillips, L.W. Swift \& C.R. Blinn, "Best management practices for riparian areas", Lewis Publishers, CRC Press LLC, 2000, pp. 273-286.

[33] M. P. Álvarez, "Evaluación de la capacidad de carga: una herramienta para el manejo y la conservación de los sitios patrimoniales", Canto Rodado, vol. 5, no 5, pp. 213-240, 2010.

[34] Y.C. Rodríguez y O.G. Zúñiga, "Estudio de capacidad de carga turística Reserva Natural Absoluta Cabo Blanco periodo 2012-2013", Programa de Voluntariado, Universidad de Costa Rica, Costa Rica, 2013, pp 27.

[35] J. A. Núñez-Castro, "Capacidad de carga turística en senderos del Parque Ecológico Chipinque A.B.C., San Pedro Garza García, Nuevo León, México", Tesis, Universidad Autónoma de Baja California Sur, México, p. 42, 2018.

[36] G. Perruolo y C. Camargo, "Estimación de la capacidad de carga turística en el área Chorro El Indio, estado Táchira, Venezuela", Revista Colombiana de Geografía, vol. 26, no 2, pp. 77-90, noviembre, 2017.

[37] I. Dias, N. Körössy y V.F. Selva, “Determinación de la capacidad de carga turística: El caso de Playa de Tamandaré, Pernambuco, Brasil", Estudios y perspectivas en turismo, vol. 21, no 6, pp. 1630-1645, 2012.

[38] W.T. Barbosa y N. Sáenz, "Diseño de la interpretación ambiental y análisis de capacidad de carga turística del sendero colonial del Parque Natural Chicaque (San Antonio Del Tequendama-Cundinamarca)", Tesis de Ingeniería Forestal, Universidad Distrital Francisco José Caldas, Bogotá, Colombia, pp 119, 2017.

[39] J. Hernández, "Capacidad de carga turística en el sendero de la Finca Pedagógica San Francisco de la ciudad de Huaca para la conservación de los recursos naturales turísticos que se encuentran en el mismo", Tesis en Ingeniería en Turismo y Ecoturismo, Universidad Politécnica Estatal del Carchi, Ecuador, pp. 96, 2019.

[40] A. M. Yoc-Aguilar, "Parque Ecoturístico Refugio del Quetzal, San Rafael Pie de la Cuesta, San Marcos", Tesis, Universidad San Carlos de Guatemala, Guatemala, pp. 77-81, 2015. 